\title{
Unilateral application of an external pneumatic compression therapy improves skin blood flow and vascular reactivity bilaterally
}

\author{
Jeffrey S Martin Corresp.. 1, 2, Allison M Martin ${ }^{1}$, Petey W Mumford ${ }^{2}$, Lorena P Salom ${ }^{2}$, Angelique N Moore ${ }^{1}$, \\ David D Pascoe ${ }^{2}$ \\ 1 Department of Biomedical Sciences, Edward Via College of Osteopathic Medicine - Auburn Campus, Auburn, United States \\ 2 School of Kinesiology, Auburn University, Auburn, United States \\ Corresponding Author: Jeffrey S Martin \\ Email address: jmartin@auburn.vcom.edu
}

Background: We sought to determine the effects of unilateral lower-limb external pneumatic compression (EPC) on bilateral lower-limb vascular reactivity and skin blood flow.

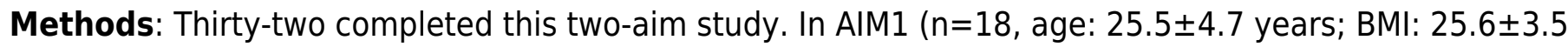
$\mathrm{kg} / \mathrm{m}^{2}$ ), bilateral femoral artery blood flow and reactivity (flow mediated dilation [FMD]) measurements were performed via ultrasonography at baseline (PRE) and immediately following 30-min of unilateral EPC treatment (POST). AIM2 ( $\mathrm{n}=14$, age: $25.9 \pm 4.5$; BMI: $27.2 \pm 2.7 \mathrm{~kg} / \mathrm{m}^{2}$ ) involved 30 -min unilateral EPC $(n=7)$ or sham $(n=7)$ treatment with thermographic bilateral lower-limb mean skin temperature (MST) measurements at baseline, at 15-min of treatment (T15) at 0, 30 and 60-min (R0, R30, R60) following treatment.

Results: Comparative data herein are presented as mean $\pm 95 \%$ confidence interval. AIM1: No significant effects on total reactive hyperemia blood flow were observed for the treated (i.e., compressed) or untreated (i.e., non-compressed) leg. A significant effect of time, but no time*leg interaction, was observed for relative FMD indicating higher reactivity bilaterally with unilateral EPC treatment (FMD: $+0.41 \pm 0.09 \%$ across both legs; $p<0.05)$. AIM2: Unilateral EPC treatment was associated with significant increases in whole-leg MST from baseline during (T15: $+0.63 \pm 0.56{ }^{\circ} \mathrm{C}$ in the visible untreated/contralateral leg, $\mathrm{p}<0.025)$ and immediately following treatment (i.e., R0) in both treated $\left(+1.53 \pm 0.59^{\circ} \mathrm{C}\right)$ and untreated $\left(+0.60 \pm 0.45^{\circ} \mathrm{C}\right)$ legs $(\mathrm{p}<0.0125)$. Across both legs, MST remained elevated with EPC at 30 -min post-treatment $\left(+0.60 \pm 0.45{ }^{\circ} \mathrm{C} ; \mathrm{p}<0.0167\right)$ but not at 60 -min post $\left(+0.27 \pm 0.46{ }^{\circ} \mathrm{C} ; \mathrm{p}=0.165\right)$. Sham treatment was associated with a significant increase in the treated leg immediately post-treatment $\left(+1.12 \pm 0.31{ }^{\circ} \mathrm{C} ; \mathrm{p}<0.0167\right)$, but not in the untreated leg $(-0.27$ $\left.\pm 0.12^{\circ} \mathrm{C}\right) . \mathrm{MST}$ in neither the treated or untreated leg were increased relative to baseline at R30 or R60 ( $p>0.05)$. Finally, during treatment and at all post-treatment time points (i.e., R0, R30 and R60), independent of treatment group (EPC vs. sham), there was a significant effect of region. The maximum increase in MST was observed at the R0 time point and was significantly $(p<0.05)$ larger in the thigh region $\left(+1.02 \pm 0.31{ }^{\circ} \mathrm{C}\right)$ than the lower-leg $\left(+0.47 \pm 0.29^{\circ} \mathrm{C}\right)$ region. However, similar rates of MST decline from R0 in the thigh and lower leg regions were observed at the R30 and R60 time points.

Discussion: Unilateral EPC may be an effective intervention for increasing skin blood flow and/or peripheral conduit vascular reactivity in the contralateral limb. While EPC was effective in increasing whole-leg MST bilaterally, there appeared to be a more robust response in the thigh compared to the lower-leg. Thus, proximity along the leg may be an important consideration in prospective treatment strategies. 
1 Unilateral application of an external pneumatic compression therapy

2 improves skin blood flow and vascular reactivity bilaterally

3

4 Jeffrey S. Martin ${ }^{1,2}$, Allison M. Martin ${ }^{1}$, Petey W. Mumford ${ }^{2}$, Lorena P. Salom ${ }^{2}$, Angelique N.

5 Moore $^{1}$, David D. Pascoe ${ }^{2}$

6

$7 \quad{ }^{1}$ Department of Biomedical Sciences, Edward Via College of Osteopathic Medicine-Auburn

8 Campus, Auburn, AL USA 36832

$9{ }^{2}$ School of Kinesiology, Auburn University, Auburn, AL USA 36849;

10

11 Corresponding Author:

12 Jeffrey S. Martin, Ph.D.

13 Department of Biomedical Sciences

14 Edward Via College of Osteopathic Medicine - Auburn Campus

15910 S. Donahue Drive

16 Auburn, AL USA 36832

17 Email: jmartin@auburn.vcom.edu 
18 Abstract

19 Background: We sought to determine the effects of unilateral lower-limb external pneumatic compression (EPC) on bilateral lower-limb vascular reactivity and skin blood flow.

Methods: Thirty-two completed this two-aim study. In AIM1 (n=18, age: 25.5 \pm 4.7 years; BMI:

$25.6 \pm 3.5 \mathrm{~kg} / \mathrm{m}^{2}$ ), bilateral femoral artery blood flow and reactivity (flow mediated dilation [FMD]) measurements were performed via ultrasonography at baseline (PRE) and immediately following 30-min of unilateral EPC treatment (POST). AIM2 (n=14, age: 25.9 \pm 4.5 ; BMI: $\left.27.2 \pm 2.7 \mathrm{~kg} / \mathrm{m}^{2}\right)$ involved 30-min unilateral EPC $(\mathrm{n}=7)$ or sham $(\mathrm{n}=7)$ treatment with thermographic bilateral lower-limb mean skin temperature (MST) measurements at baseline, at 15-min of treatment (T15) at 0, 30 and 60-min (R0, R30, R60) following treatment.

Results: Comparative data herein are presented as mean $\pm 95 \%$ confidence interval. AIM1: No significant effects on total reactive hyperemia blood flow were observed for the treated (i.e., compressed) or untreated (i.e., non-compressed) leg. A significant effect of time, but no time*leg interaction, was observed for relative FMD indicating higher reactivity bilaterally with unilateral EPC treatment (FMD: $+0.41 \pm 0.09 \%$ across both legs; $<<0.05)$. AIM2: Unilateral EPC treatment was associated with significant increases in whole-leg MST from baseline during $\left(\mathrm{T} 15:+0.63 \pm 0.56{ }^{\circ} \mathrm{C}\right.$ in the visible untreated/contralateral leg, $\left.\mathrm{p}<0.025\right)$ and immediately following treatment (i.e., R0) in both treated $\left(+1.53 \pm 0.59{ }^{\circ} \mathrm{C}\right)$ and untreated $\left(+0.60 \pm 0.45^{\circ} \mathrm{C}\right)$ legs $(\mathrm{p}<0.0125)$. Across both legs, MST remained elevated with EPC at 30-min post-treatment $\left(+0.60 \pm 0.45{ }^{\circ} \mathrm{C} ; \mathrm{p}<0.0167\right)$ but not at $60-\min$ post $\left(+0.27 \pm 0.46{ }^{\circ} \mathrm{C} ; \mathrm{p}=0.165\right)$. Sham treatment was associated with a significant increase in the treated leg immediately post-treatment $(+1.12 \pm$ $\left.0.31{ }^{\circ} \mathrm{C} ; \mathrm{p}<0.0167\right)$, but not in the untreated leg $\left(-0.27 \pm 0.12{ }^{\circ} \mathrm{C}\right)$. MST in neither the treated or untreated leg were increased relative to baseline at R30 or R60 ( $>>0.05)$. Finally, during 
41 treatment and at all post-treatment time points (i.e., R0, R30 and R60), independent of treatment

42 group (EPC vs. sham), there was a significant effect of region. The maximum increase in MST

43 was observed at the R0 time point and was significantly $(\mathrm{p}<0.05)$ larger in the thigh region

$44\left(+1.02 \pm 0.31^{\circ} \mathrm{C}\right)$ than the lower-leg $\left(+0.47 \pm 0.29^{\circ} \mathrm{C}\right)$ region. However, similar rates of MST

45 decline from R0 in the thigh and lower leg regions were observed at the R30 and R60 time

46 points.

47 Discussion: Unilateral EPC may be an effective intervention for increasing skin blood flow

48 and/or peripheral conduit vascular reactivity in the contralateral limb. While EPC was effective

49 in increasing whole-leg MST bilaterally, there appeared to be a more robust response in the thigh

50 compared to the lower-leg. Thus, proximity along the leg may be an important consideration in

51 prospective treatment strategies. 


\section{Introduction}

Chronic wound care therapies often target improvements in local blood flow. However,

54 one of the difficulties in the treatment of chronic wounds is the pain and complication associated with direct wound contact. Interventions aiming to circumvent this problem that, in part, aim to improve blood flow and healing in chronic wounds include vacuum-assisted closure, local tissue warming and non-contact radiant bandages. However, the potential for a whole-leg, dynamic, external pneumatic compression (EPC) therapy applied to a non-affected limb has not been explored. We have previously demonstrated that a single treatment with a low pressure $(\leq 100$ mmHg) EPC device (NormaTec Pro, NormaTec, Newton Center, MA, USA) acutely improves reactivity in the peripheral conduit and resistance vasculature of both compressed (i.e. legs) and non-compressed (i.e. arms) limbs (Martin et al. 2015a). Moreover, we have shown that local concentration of the stable metabolites of nitric oxide (NO) are increased in skeletal muscle biopsy samples following a 1-h bout of EPC (Kephart et al. 2015). Nevertheless, it has yet to be determined what effect unilateral EPC treatment would have on the contralateral limb. Recently, Credeur et al showed that unilateral treatment for 1-h with intermittent pneumatic compression (IPC) at target inflation pressures of $120 \mathrm{mmHg}$ in spinal cord injury patients acutely increases posterior-tibial artery reactivity in the treated (compressed) leg, but not the untreated leg (2017). However, the aforementioned EPC device used in our prior investigations differs markedly from IPC as it is more dynamic (peristaltic compression pattern), utilizes a different duty cycle, contains additional inflation chambers and encompasses the entire leg. Given the need for noncontact and easily accessible interventions that can effectively improve blood flow and endothelial health to facilitate chronic wound healing, as a first step, we sought to determine the effect of dynamic, whole-leg EPC applied to a single leg on vascular reactivity and blood flow in 
75 both legs in a healthy population. We hypothesized that unilateral EPC treatment would

76 effectively improve vascular reactivity, as determined by flow mediated dilation (FMD; AIM1),

77 and skin microcirculation, as determined by dynamic infrared thermography mean skin

78 temperature (MST; AIM2), in both the treated (i.e., compressed/covered) and untreated (i.e.,

non-compressed/non-covered) legs. In addition, we hypothesized that the improvements in skin

80 blood flow in the limbs would be global (i.e., not segmental) in nature.

\section{Materials \& Methods}

\section{Participants}

Twenty $(\mathrm{N}=20)$ and fourteen $(\mathrm{N}=14)$ apparently healthy persons were recruited from the

local community to participate in AIM1 and AIM2 of this study, respectively. All procedures

herein were approved by Auburn University’s Institutional Review Board (Project \#15-017

MR1502) and conformed to the standards set by the latest revision of the Declaration of

Helsinki. Written informed consent was obtained from all participants prior to their voluntary participation in the study.

\section{Study Design}

For AIM1, twenty persons (10 females, 10 males) reported for a single visit which was comprised of baseline bilateral blood pressure and popliteal artery FMD measurements, 30-min of unilateral EPC application with target inflation pressures of $70 \mathrm{mmHg}$, and a second set of bilateral FMD measurements 30-min after completion of the EPC treatment (POST). Bilateral blood pressure measures were employed to identify any significant between arm variability

96 (defined as $\geq 10 \mathrm{mmHg}$ for systolic or diastolic blood pressure) that may confound the results

97 (Martin et al. 2015b). In AIM1, two persons were identified as having significant between arm 
98 blood pressure variability and were excluded from the study. Thus, the total number of

99 participants for AIM1 was eighteen (9 females, 9 males).

$100 \quad A I M 2$

101 For AIM2, fourteen participants (4 females, 10 males) reported for a single visit which

102 was comprised of baseline bilateral blood pressure and leg thermography measurements,

103 randomization to either 30-min of unilateral EPC treatment with target inflation pressures of 70

$104 \mathrm{mmHg}(\mathrm{n}=7)$ or a 30-min sham treatment with unilateral positioning of an EPC "leg sleeve" with

105 no inflation/compression $(\mathrm{n}=7)$, and 60-min of post-EPC/sham thermography measurements.

106 Medical histories were assessed to confirm absence of medical conditions that might interfere

107 with the intervention(s) or outcomes (e.g. orthopedic injuries/surgeries, neurological issues,

108 diabetes, etc.). All participants were found to have regular thermal patterns in and between the

109 two legs per preliminary imaging and were free from significant between arm blood pressure

110 differences.

111 We have observed (informally) that the ultrasound/FMD procedure creates a slight,

112 transient (<5 minutes) increase in skin surface temperature specific to the area of contact. Thus,

113 separate trials were performed for each aim of the study.

\section{Procedures}

115 For both aims, all participants were instructed to abstain from exercise and alcohol for

116 24-h and from caffeine for 12-h. Additionally, all participants reported to the laboratory at least

117 2-h post-prandial and, in order to control for any potential diurnal variation, at the same time of 118 day.

119 Brachial artery blood pressure

120 For both aims, upon reporting to the laboratory, height and weight was measured using a

121 digital scale with height rod. Thereafter, following 15-min of supine rest, heart rate and brachial 
122 artery systolic and diastolic blood pressure measurements were made at both arms using an

123 automated oscillometric device (OMRON BP785, Omron Corporation, Kyoto, Japan). The

124 average of the measurements at the left and right arm were recorded for characterization of blood

125 pressure.

126 Flow Mediated Dilation

127 In AIM1, following baseline heart rate and blood pressure measurement, popliteal artery

128 FMD was measured in both legs at baseline (i.e. PRE) and 30-min after (i.e., POST) unilateral

129 EPC application. The order of FMD measurement side (i.e. left or right leg) at baseline was

130 randomized for each subject, though that order was repeated for post-EPC measures.

131 FMD was used to determine endothelial-dependent reactivity in the popliteal arteries using

132 high resolution ultrasound (GE Logiq S7 R2 Expert) with a 3 to $12 \mathrm{MHz}$ multi-frequency linear

133 phase array transducer. In addition, the reactive hyperemia response (i.e. peak and total reactive

134 hyperemia blood flow) during FMD procedures was determined for evaluation of microvascular

135 (i.e. arterioles and capillaries) reactivity. In brief, each artery was imaged longitudinally with the

136 transducer placed $3-8 \mathrm{~cm}$ below the popliteal fossa and held manually in the same position for

137 the duration of the measurements. Simultaneous measurement of artery diameter and blood

138 velocity was performed using duplex mode imaging (B-mode and Doppler) and video was

139 captured through a digital interface at 30 frames/second with real time analysis (FMD Studio

140 v2.8, QUIPU, Pisa, Italy). Resting measurements were captured for 1-min, reactive hyperemia

141 was produced by inflating a cuff placed on the calf for 5-min at $200 \mathrm{mmHg}$, and, after cuff

142 release, measurements were made for another 3-min. Vessel diameters were determined via

143 automatic edge detection software (FMD Studio) measuring the distance between the near and

144 far wall of the intima. Blood velocity was determined via selection of a region of interest around

145 the Doppler waveform and a trace of the velocity-time integral was used to calculate mean 
146 velocity for each cardiac cycle. Shear rate was calculated as [( $4 *$ time averaged mean

147 velocity)/vessel diameter] and mean blood flow was calculated as $\left[\Pi *(\text { diameter } / 2)^{2} *\right.$ time

148 average mean velocity *60].

149 FMD was calculated as absolute (aFMD; in $\mathrm{mm}$ ) and relative (\%FMD) peak change in

150 artery diameter in response to the hyperemic stimulus. Because dilation also depends on the

151 resultant reactive hyperemia shear rate stimulus, normalized FMD (nFMD) was calculated as the

152 ratio of $\%$ FMD to the shear rate area under the curve (AUC) to the time at which the maximal

153 diameter was observed (100*\%FMD/shear rate AUC). Finally, peak reactive hyperemia blood

154 flow and total reactive hyperemia blood flow AUC were calculated from observed 5-s averages

155 during the reactive hyperemia period. AUC was determined using the sum of trapezoids method

156 after baseline values were subtracted.

157 Thermography assessment of skin blood flow

158 In AIM2, following 15-min of rest for equilibration to room/position conditions, infrared

159 images of the anterior aspect of the legs were taken using an infrared thermal imaging system

160 camera and processor (Computerized Thermal Imaging Inc., Ogden, UT, USA). Regions of

161 interest included the anterior thigh and lower leg. Leg region MST was determined from the

162 processor program that provides the average value of the pixels within the regions of interest.

163 Images were taken within the thermal lab where the temperature was maintained at $21 \pm 1.1^{\circ} \mathrm{C}$

164 (thermoneutral environment) and emissivity was set at 0.98. Images were taken at baseline, at

165 the mid-point of EPC/sham treatment (T15), immediately post-EPC/sham treatment (R0), and at

166 30- (R30) and 60-min (R60) following EPC/sham treatment. All participants wore gym shorts

167 and non-constrictive undergarments appropriate for complete visualization of the thighs. A

168 representative thermography image is presented in Fig. 1. 


\section{External pneumatic compression (EPC) and sham}

For both AIM1 and AIM2, a dynamic, sequential EPC device (NormaTec Pro, Newton Center, MA, USA) was applied for 30-min. Briefly, the EPC device utilizes "leg sleeves” which contain five circumferential inflatable chambers (arranged linearly) encompassing the leg from the feet to the hip/groin. The "leg sleeves" are connected to an automated pneumatic pump at which duration of treatment and target inflation pressures can be controlled. In this study, we chose to use target inflation pressures of $\sim 70 \mathrm{mmHg}$ for each chamber as we've previously observed acute improvements in vascular reactivity with bilateral treatment at this target inflation pressure (Martin et al. 2015a). Herein, only one leg sleeve was applied (right leg) while the contralateral leg (left) was uncovered. At the onset of the EPC treatment, all zones in the "leg sleeve" are pre-inflated to form fit the participant's legs while applying minimal pressure $(\sim 10$ $\mathrm{mmHg}$ ). Thereafter, the distalmost chamber (which covers from the high ankle to toes) inflated to approximately $70 \mathrm{mmHg}$ while the remaining zones were not inflated. For 30 -s, this chamber "pulsed" after which the pressure was held constant at $70 \mathrm{mmHg}$ and the same process occurred in the zone above (calves) for another 30 -s. Following this $30-\mathrm{s}$, the ankle zone deflated completely, the calf remained at a constant pressure of approximately $70 \mathrm{mmHg}$, and the process occurred in the next highest zone (high claves/lower thighs). This continued until the highest zone ( $5^{\text {th }}$ zone/upper thighs) finished the process at which point all zones were completely deflated for 30-s and a full compression cycle (3-min total) was completed. This entire cycle of compression was repeated continuously over the course of the treatment session which lasted for 30-min.

In AIM2, a sham condition was also employed which consisted of 30-min application of a single EPC "leg sleeve" on the right leg, but devoid of any actual compression. A sham was 
192 included in AIM2 to assess the thermogenic effect from contact and/or attenuation of heat loss

193 from simply wearing the "leg sleeve".

194 Statistical Analysis

195 All statistical analyses were performed using SPSS v22.0 (IBM Corp., Armonk, NY, 196 USA). Prior to statistical analysis normality was confirmed using Shapiro-Wilk tests. For 197 ANOVA, if sphericity was violated a correction factor was applied to hypothesis testing. Subject 198 characteristics for both aims were compared using independent t-tests.

For AIM1 dependent variables, two-way repeated measures ANOVAs were performed with time (PRE vs. POST) and leg (treated vs. untreated) as independent variables. When a significant time*leg interaction was observed, post-hoc comparisons were made using Bonferroni corrected paired t-tests.

For AIM2, MST during treatment (i.e. T15) was analyzed using a repeated measures of analysis with time (PRE vs. T15), region (thigh vs. lower-leg), and treatment group (EPC vs. sham) as the independent variables. Given the opaque nature of the leg sleeve, leg (i.e., treated vs. untreated) was not included as an independent variable as MST during respective treatments was only evaluable in the untreated leg. MST at R0, R30 and R60 was analyzed using repeated measures ANOVA with time (PRE vs. R0, R30 and R60), leg, region, and treatment group as the independent variables. When significant interactions with time were found, post-hoc

210 comparisons were made using paired and independent t-tests as appropriate with Bonferroni

211 correction for multiple comparisons (0.05/number of comparisons). Data are presented as mean

$212 \pm$ standard deviation for summary data and as mean [95\% confidence interval (lower limit, upper 213 limit)] for comparative data. 


\section{RESULTS}

\section{Subject Characteristics}

Participant characteristics for the cohorts of subjects included in AIM1 and AIM2 are

217 presented in Table 1. In AIM1, significant between sex differences existed for height, body

218 mass, and blood pressures, but not age $(\mathrm{p}=0.211)$ and BMI $(\mathrm{p}=0.123)$. In AIM2, no significant

219 between condition differences (i.e., EPC vs. sham) were found for any characteristic ( $\mathrm{p}>0.05)$.

220 Significant between sex differences existed for height $(\mathrm{p}=0.014)$, body mass $(\mathrm{p}=0.040)$, and

221 diastolic blood pressure $(p=0.039)$, but not systolic blood pressure $(p=0.130)$, age $(p=0.457)$ or

222 BMI $(\mathrm{p}=0.203)$. However, sex was not found to be a significant covariate in either AIM1 or

223 AIM2.

224 AIM1 - Effect of unilateral EPC on bilateral vascular reactivity in the legs.

Results from popliteal artery FMD assessment are presented in Table 2 and Fig. 2. No

main effects (time or treatment) or interaction was observed for resting popliteal artery diameter or blood flow (Table $2 ; \mathrm{p}>0.05$ for all). With respect to the reactive hyperemia response, a significant main effect of time, but no main effect of leg or time*leg interaction, was observed for both maximum blood flow (Fig 2A) and shear rate AUC to peak dilation $(\mathrm{p}<0.010$; Table 2). Independent of leg (collapsing across treated and untreated legs), maximum reactive hyperemia blood flow and shear rate AUC at the POST time point were decreased relative to baseline (i.e., PRE) by $39.3 \mathrm{~mL} / \mathrm{min}[-0.9,-77.8]$ and 467 a.u. [-734, -200], respectively. No main effects or interactions were observed for total reactive hyperemia blood flow (Fig 2B).

A significant effect of time $(\mathrm{p}<0.001)$, but no main effect of leg or time*leg interaction, was observed for aFMD and pFMD. Independent of leg, both aFMD $(+0.022 \mathrm{~mm}[+0.009$, $+0.035]$; Table 2$)$ and pFMD $(+0.41 \%[+0.14,+0.68]$; Fig. $2 \mathrm{C})$ were significantly higher at the POST time point relative to PRE. For nFMD, significant main effects of time and leg were 
238 observed, but no interaction (Fig. 2D). Independent of time (collapsing across PRE and POST

239 time points), $\mathrm{nFMD}$ was significantly higher in the treated leg $(+0.31[+0.10,+0.53])$ and

240 independent of leg, nFMD was significantly higher at POST $(+0.57[+0.39,+0.80])$.

241 AIM2 - Effect of unilateral EPC on bilateral leg skin temperature

Baseline (PRE) values for regional and average MST in each leg for each treatment group

243

244

245

246

247

248

249

250

251

252

253

254

255

256

257

258

259

260

261

are presented in Table 3. For during treatment $(\mathrm{T} 15)$, significant time*treatment $(\mathrm{p}=0.003)$ and time*region $(\mathrm{p}=0.031)$ interactions were observed. Across both regions, change in MST at T15 was significantly greater in the untreated leg with EPC compared to sham $\left(+0.82{ }^{\circ} \mathrm{C}[+0.34\right.$, +1.30]; Fig 3A). MST in the untreated leg was significantly elevated relative to baseline with EPC treatment EPC and significantly reduced relative to baseline with sham in the untreated leg. Across both treatments, change in MST was significantly greater in the thigh region compared to the lower-leg region $\left(+0.32{ }^{\circ} \mathrm{C}[+0.13,+0.51]\right.$; Fig 3B $)$. However, collapsing across both treatments, neither the thigh $(p=0.051)$ or lower-leg $(p=0.731)$ region were significantly different from baseline.

For $\mathrm{R} 0$, significant region*time $(\mathrm{p}<0.001)$ and treatment*leg*time $(\mathrm{p}=0.032)$ interactions were observed. Figure 3D illustrates the effect of region where, when collapsing across both treatments and legs, MST was significantly elevated from baseline at R0 at both regions $(\mathrm{p}<0.001$ and $\mathrm{p}=0.003$ for thigh and lower-leg, respectively) though the increase was significantly greater in the thigh compared to the lower-leg $\left(+0.55^{\circ} \mathrm{C}[+0.40,+0.70] ; \mathrm{p}<0.001\right)$.

With respect to the treatment*leg*time interaction (illustrated in Fig. 3C), independent of region, EPC treatment was associated with a significant increase in MST in both the treated leg $(\mathrm{p}<0.001)$ and untreated leg $(\mathrm{p}=0.002)$ at R0 whereas MST was increased with sham in the treated leg $(p<0.001)$ but decreased in the untreated leg $(p=0.002)$. Change in MST at R0 in the treated leg with EPC treatment was significantly higher than in the untreated leg with EPC 
262 treatment $\left(+0.94{ }^{\circ} \mathrm{C}[+0.56,+1.31] ; \mathrm{p}<0.001\right)$ and the untreated leg with sham treatment $(+1.81$

$\left.263{ }^{\circ} \mathrm{C}[+1.24,+2.37] ; \mathrm{p}<0.001\right)$, but not the treated leg with sham treatment $\left(+0.42{ }^{\circ} \mathrm{C}[-1.05\right.$,

$264+0.21] ; p=0.076)$. Change in MST at R0 in the treated leg with sham was significantly higher

265 than in both the untreated leg with EPC $(+0.52[-0.03,+1.07] ; p=0.012)$ and the untreated leg

266 with sham $\left(+1.39{ }^{\circ} \mathrm{C}[+1.12,+1.65] ; \mathrm{p}<0.001\right)$. Finally, in the untreated leg, change in MST at

267 R0 was greater with EPC treatment compared to sham treatment $\left(+0.87^{\circ} \mathrm{C}[+0.35,+1.38]\right.$;

$268 \mathrm{p}<0.001)$

For the R30 time point, significant treatment*time $(p<0.001)$, leg*time $(p=0.001)$ and

270

271

272

273

274

275

276

277

278

279

280

281

282

283

region*time $(\mathrm{p}<0.001)$ interactions were observed. Across both regions and legs, MST was

significantly elevated relative to baseline with EPC treatment $(\mathrm{p}<0.001)$ and significantly

reduced relative to baseline with sham treatment $(\mathrm{p}=0.003$; Fig. $4 \mathrm{~A})$. Across both treatments and

legs, thigh MST was significantly elevated relative to baseline $(\mathrm{p}<0.001)$, but not lower-leg MST

$(p=0.558$; Fig. 4B). Finally, across both regions and treatments, treated $(p=0.034)$ and untreated

$(p=0.873)$ leg MST were no longer significantly elevated relative to baseline (Fig. 4C).

Similar to the R30 time point, at the R60 time point, significant treatment*time

$(p=0.002)$, leg*time $(p=0.008)$ and region*time $(p<0.001)$ interactions were observed. Across

both regions and legs, MST was no longer significantly elevated relative to baseline with EPC

treatment $(\mathrm{p}=0.051)$, but remained significantly reduced relative to baseline with sham treatment

$(\mathrm{p}<0.001$; Fig. 4D. Across both treatments and legs, thigh MST was also no longer significantly

elevated relative to baseline $(\mathrm{p}=0.101)$, but now significantly reduced relative to baseline in the

lower-leg MST ( $<<0.001$; Fig. 4E). Finally, across both regions and treatments, treated leg

$(p=0.492)$ and untreated leg $(p=0.075)$ MST remained unchanged relative to baseline (Fig. 4F).

PeerJ reviewing PDF | (2018:02:24319:1:1:NEW 26 Apr 2018) 


\section{Discussion}

The primary findings of the present study are as follows; 1) 30-min of unilateral EPC is associated with increased popliteal artery FMD in both the treated and untreated leg post-EPC, 2)

MST during treatment is significantly increased relative to baseline in the untreated leg with EPC

immediately following EPC treatment, but only in the treated leg with sham treatment, 4) the increase in MST in the treated leg was more transient with sham treatment compared to EPC treatment, and 5) independent of other factors, the thigh region was associated with more robust and sustained increases in MST compared to the lower-leg.

Regarding AIM1 of the present investigation, the bilateral improvement in popliteal artery FMD with unilateral treatment is similar to our previous observations of improved popliteal (compressed leg) and brachial artery (non-compressed arm) FMD with 1-h of bilateral leg EPC treatment (Martin et al. 2015a). Herein, we extend our previous findings by demonstrating that 1) unilateral EPC improves vascular reactivity in the treated and untreated (contralateral) leg, 2) 30-min of treatment, compared to 1-h, is sufficient for improvement of peripheral conduit reactivity, and 3) FMD is improved for at least 30-min following treatment. Improved FMD within the treated leg could be explained by markedly altered hemodynamic shear stress, and resultant local nitric oxide (NO) bioavailability, during EPC treatment (Martin et al. 2016), but this is an unlikely mechanism in the contralateral leg. However, a single 1-h bout of IPC has been shown to affect systemic measures of NO as well as non-local vascular reactivity (Rifkind et al. 2014). While IPC does differ markedly from the EPC therapy used herein, it is not unreasonable to assume that other external compression stimuli can modulate 306 changes in non-local and/or systemic NO bioavailability. We have also previously posited that 
307 EPC-mediated myokine (e.g., IL-6) release may potentiate systemic NO bioavailability

308 (Fisslthaler \& Fleming 2009; Horman et al. 2008; Pedersen \& Febbraio 2008), but this remains

309 unknown. Moreover, while nitric oxide bioavailability may play a role in the observed acute

310 FMD increase in the untreated leg, a decrease in sympathetic nervous system (SNS) efferent

311 activity may also be involved in the observed response with EPC treatment (Dyson et al. 2006;

312 Hijmering et al. 2002; Lind et al. 2002). Indeed, the pressor effect from static compression on

313 the lower limb(s), at a range of pressures $(30-90 \mathrm{mmHg})$, and with single limb compression, has

314 been shown previously and is abolished by epidural anesthesia (Williamson et al. 1994).

315 Moreover, we have previously observed acute increases in peripheral (brachial) and central

316 (aortic) blood pressure for at least 10-min following bilateral EPC treatment (Vincent-Horta et al.

317 2015). Thus, it is possible that the arterial baroreflex may modulate sympathetic outflow in

318 response to acute EPC treatment. However, the role of the sympathetic nervous system in the

319 acute FMD response to EPC requires further, specific, investigation.

320 In regards to AIM2 of the present investigation, thermography measures temperature

321 changes within the cutaneous microcirculation plexus that are modulated by skin blood flow

322 perfusion. As such, infrared imaging provides a non-contact thermal map of the skin surface

323 area that reveals both spatial and temporal changes that occur in the regional temperature

324 distribution (Pascoe et al. 2012). Notably, although infrared imaging provides accurate thermal

325 measures of skin surface temperatures that are responsive to blood flow alterations, it cannot

326 quantify blood flow itself.

327 In the treated legs, immediately following EPC/sham treatment (i.e., R0) a similar,

328 significant rise in temperature of greater than $1^{\circ} \mathrm{C}$ was observed with both $\mathrm{EPC}\left(+1.53{ }^{\circ} \mathrm{C}\right)$ and

329 sham $\left(+1.12^{\circ} \mathrm{C}\right)$. The EPC "leg sleeve" is a non-breathable, thick material for which conductive 
330 contact did not allow for heat transfers (Havenith 1999), likely mediating a significant proportion

331 of the increase in MST of the treated leg with both EPC and sham. Indeed, while the change in

332 MST was greater in the treated leg with EPC compared to sham $(+0.42)$ it was not a statistically

333 significant difference $(\mathrm{p}=0.073)$. However, in the untreated leg, immediately following

334 completion of sham/EPC treatment (i.e. R0), MST was significantly elevated relative to baseline

335 with EPC $\left(+0.60^{\circ} \mathrm{C}\right)$, but significantly decreased relative to baseline with sham $\left(-0.27^{\circ} \mathrm{C}\right)$

336 illustrating a markedly different response to the two treatments. This was similar to what was

337 observed during treatment (i.e. T15), where there was a significant increase across both regions

338 in the untreated leg with EPC $\left(+0.83^{\circ} \mathrm{C}\right)$, but a significant decrease with sham $\left(-0.20{ }^{\circ} \mathrm{C}\right)$. Thus,

339 it would appear that during and immediately following unilateral treatment, the addition of a

340 pneumatic compression stimulus not only prevents a decrease in MST in the contralateral limb,

341 but actually promotes a significant increase. Although skin sympathetic nerve activity (Wallin et

342 al. 1975) and sweat rate (Wilson et al. 2001) do not change with the aforementioned arterial

343 baroreflex, changes in cutaneous vascular conductance have been observed (Crandall et al. 1996;

344 Kellogg et al. 1990; Tripathi \& Nadel 1986). Regardless, one explanation could simply be the

345 distribution, or re-distribution, of heat and/or blood in the vascular space (Arndt et al. 1985).

346 Indeed, the higher MST in the contralateral leg with EPC, but not sham, during and immediately

347 following treatment could be due to heat added to the system from the dynamic compression

348 stimulus (i.e., tissue deformation) and/or compression itself mobilizing fluid(s) including, but not

349 limited to, a proportion of venous and arterial flow.

350 Differential responses between treatments (EPC vs. sham) were also observed during the

351 recovery period from EPC/sham treatment (i.e., R30, R60). Indeed, 30-min following

352 completion of unilateral treatment, independent of leg, MST remained elevated respective to 
353 baseline with EPC and was actually reduced relative to baseline with sham treatment. In

354 addition, 60-min following treatment, independent of leg, while MST had returned to baseline

355 with EPC treatment, it remained reduced relative to baseline with sham. The absence of a

356 significant interaction with the factor of leg (treated vs. untreated) suggests an additional effect

357 of unilateral dynamic compression on the bilateral thermal recovery response. In the treated leg,

358 transmural pressure changes in the vasculature of the compressed tissue are likely associated

359 with an auto-regulatory (i.e., myogenic) response which may augment skin blood flow and MST.

360 Importantly, this effect has been shown to persist for hours following pressure application

361 (Bochmann et al. 2005). However, devoid of a compression stimulus, this is unlikely to be a

362 major contributor to the contralateral limb. Thermal control over cutaneous vascular responses

363 has not been fully elucidated but have affirmed roles for temperature sensitive afferent neurons,

364 sympathetic/parasympathetic controls and NO mediated vasodilation (Kellogg 2006) which may

365 have, in some capacity, contributed to our observations. While speculative, acute massage, to

366 which lower limb EPC is often compared, has been shown to elicit a decrease in sympathetic

367 nervous system efferent activity and an increase in parasympathetic nervous system activity

368 (Diego \& Field 2009). Moreover, twenty 40-min sessions of massage therapy over the course of

369 4-weeks has been shown to decrease autonomic nerve conduction latency and amplitude (Lee et

370 al. 2011). However, future studies are warranted with respect to autonomic nervous system

371 modulation and its role in local and non-local responses to EPC treatment.

372 Several findings herein would appear to be of interest from a clinical standpoint. First,

373 acute improvements in bilateral popliteal artery FMD would suggest improved peripheral

374 vascular reactivity which may potentiate greater blood flow and wound healing (Guo \& Dipietro

375 2010). In addition, improved endothelium dependent and independent vascular reactivity may 
376 decrease the risk for diabetic foot ulcer development (Dinh et al. 2012). Secondly, although we

377 did not observe any change following the EPC condition in peak or total reactive hyperemia

378 assessed with ultrasonography during the FMD procedure, this is similar to our previous findings

379 with EPC (Martin et al. 2015a). Notably, in that study, despite no changes in reactive hyperemia

380 assessed via ultrasonography, we did identify an acute increase in peak reactive hyperemia blood

381 flow responses immediately following an EPC condition when assessed via venous occlusion

382 plethysmography. Finally, local blood flow improvements may mitigate negative ischemic,

383 inflammatory, oxidative, and bacterial associated factors in wound healing (Guo \& Dipietro

384 2010; Hopf et al. 1997; Kurz et al. 1996; Mustoe 2004). Herein, we observed marked increases

385 in MST, a surrogate of skin blood flow, in the contralateral leg during and for at least 30-min

386 following unilateral EPC treatment. Thus, non-contact treatment via compression of the

387 unaffected limb may be a viable strategy to improve skin blood flow during wound healing.

388 Importantly, at all time points (during and after treatment) significant effects of region were

389 observed with a more robust increase in thigh MST being observed initially with a subsequently

390 similar rate of decline over the recovery period. Thus, the regional nature of the responses needs

391 to be considered. In addition, from an experimental model perspective, our findings suggest

392 treatment differences may be disguised when using a one leg (control) model in which the

393 untreated leg is altered by the intervention. In our case, the increased temperatures in the

394 "control" leg lessened the calculated statistical effect between legs. Finally, we used target

395 inflation pressures of $\sim 70 \mathrm{mmHg}$ only for the EPC intervention and the response to higher or

396 lower target inflation pressure(s) may vary. 


\section{Conclusions}

398 Unilateral, dynamic, whole-leg EPC treatment increases bilateral vascular reactivity and 399 MST. With respect to MST, it is important to recognize that although there is a greater and more 400 persistent effect in the treated leg with MST, single leg treatment will also provide a clinically

401 significant increase in the contralateral limb. Moreover, EPC treatments of the leg were strongly 402 influenced by the intervention response in the thigh, and to a lesser extent the lower leg. From a 403 clinical perspective, our results suggest that EPC could be utilized to increase blood flow in an 404 afflicted limb (e.g. injury, peripheral vascular disease, etc.) with contralateral application, 405 particularly when the target area is more proximal in the leg.

\section{Acknowledgements}

407 The authors wish to thank the participants for their compliance and for devoting their

408 time to this study. 
409 References

410 Arndt JO, Hock A, Stanton-Hicks M, and Stuhmeier KD. 1985. Peridural anesthesia and the 411 distribution of blood in supine humans. Anesthesiology 63:616-623.

412 Bochmann RP, Seibel W, Haase E, Hietschold V, Rodel H, and Deussen A. 2005. External compression increases forearm perfusion. J Appl Physiol (1985) 99:2337-2344.

414 10.1152/japplphysiol.00965.2004

415

416

Crandall C, Johnson J, Kosiba W, and Kellogg Jr D. 1996. Baroreceptor control of the cutaneous active vasodilator system. Journal of applied physiology 81:2192-2198.

417 Credeur DP, Vana LM, Kelley ET, Stoner L, and Dolbow DR. 2017. Effects of Intermittent

Diego MA, and Field T. 2009. Moderate pressure massage elicits a parasympathetic nervous Pneumatic Compression on Leg Vascular Function in People with Spinal Cord Injury: A Pilot Study. J Spinal Cord Med:1-9. 10.1080/10790268.2017.1360557

422

423

424

425

426

427

428

429

430

431 system response. Int J Neurosci 119:630-638. 10.1080/00207450802329605

Dinh T, Tecilazich F, Kafanas A, Doupis J, Gnardellis C, Leal E, Tellechea A, Pradhan L, Lyons TE, Giurini JM, and Veves A. 2012. Mechanisms involved in the development and healing of diabetic foot ulceration. Diabetes 61:2937-2947. 10.2337/db12-0227

Dyson KS, Shoemaker JK, and Hughson RL. 2006. Effect of acute sympathetic nervous system activation on flow-mediated dilation of brachial artery. Am J Physiol Heart Circ Physiol 290:H1446-1453. 10.1152/ajpheart.00771.2005

Fisslthaler B, and Fleming I. 2009. Activation and signaling by the AMP-activated protein kinase in endothelial cells. Circ Res 105:114-127. 10.1161/CIRCRESAHA.109.201590

Guo S, and Dipietro LA. 2010. Factors affecting wound healing. J Dent Res 89:219-229. $10.1177 / 0022034509359125$ 
432 Havenith G. 1999. Heat balance when wearing protective clothing. The Annals of occupational

433

434

435

436

437

438

439

440

441

442

443

444

445

446

447

448

449

450

451

452

453

454 hygiene 43:289-296.

Hijmering ML, Stroes ES, Olijhoek J, Hutten BA, Blankestijn PJ, and Rabelink TJ. 2002. Sympathetic activation markedly reduces endothelium-dependent, flow-mediated vasodilation. J Am Coll Cardiol 39:683-688.

Hopf HW, Hunt TK, West JM, Blomquist P, Goodson WH, 3rd, Jensen JA, Jonsson K, Paty PB, Rabkin JM, Upton RA, von Smitten K, and Whitney JD. 1997. Wound tissue oxygen tension predicts the risk of wound infection in surgical patients. Arch Surg 132:997-1004; discussion 1005.

Horman S, Morel N, Vertommen D, Hussain N, Neumann D, Beauloye C, El Najjar N, Forcet C, Viollet B, Walsh MP, Hue L, and Rider MH. 2008. AMP-activated protein kinase phosphorylates and desensitizes smooth muscle myosin light chain kinase. J Biol Chem 283:18505-18512. 10.1074/jbc.M802053200

Kellogg DL, Johnson JM, and Kosiba W. 1990. Baroreflex control of the cutaneous active vasodilator system in humans. Circulation Research 66:1420-1426.

Kellogg DL, Jr. 2006. In vivo mechanisms of cutaneous vasodilation and vasoconstriction in humans during thermoregulatory challenges. J Appl Physiol (1985) 100:1709-1718. 10.1152/japplphysiol.01071.2005

Kephart WC, Mobley CB, Fox CD, Pascoe DD, Sefton JM, Wilson TJ, Goodlett MD, Kavazis AN, Roberts MD, and Martin JS. 2015. A single bout of whole-leg, peristaltic pulse external pneumatic compression upregulates PGC-1alpha mRNA and endothelial nitric oxide sythase protein in human skeletal muscle tissue. Exp Physiol 100:852-864.

$10.1113 / \mathrm{EP} 085160$ 
455 Kurz A, Sessler DI, and Lenhardt R. 1996. Perioperative normothermia to reduce the incidence 456 of surgical-wound infection and shorten hospitalization. Study of Wound Infection and Temperature Group. N Engl J Med 334:1209-1215. 10.1056/NEJM199605093341901

458

459

460

461

462

463

464

465

466

467

468

469

470

471

472

473

474

475

476

477 flow-mediated vasodilation. Blood Press 11:22-27.

Martin JS, Borges AR, and Beck DT. 2015a. Peripheral conduit and resistance artery function are improved following a single, 1 -h bout of peristaltic pulse external pneumatic compression. Eur J Appl Physiol 115:2019-2029. 10.1007/s00421-015-3187-8

Martin JS, Borges AR, Christy JBt, and Beck DT. 2015b. Considerations for SphygmoCor radial artery pulse wave analysis: side selection and peripheral arterial blood pressure calibration. Hypertens Res 38:675-683. 10.1038/hr.2015.36

Martin JS, Kephart WC, Haun CT, McCloskey AE, Shake JJ, Mobley CB, Goodlett MD, Kavazis A, Pascoe DD, Zhang L, and Roberts MD. 2016. Impact of external pneumatic compression target inflation pressure on transcriptome-wide RNA expression in skeletal muscle. Physiol Rep 4. 10.14814/phy2.13029

Mustoe T. 2004. Understanding chronic wounds: a unifying hypothesis on their pathogenesis and implications for therapy. Am J Surg 187:65S-70S. 10.1016/S0002-9610(03)00306-4

Pascoe DD, de Weerd L, Mercer JB, Lane JE, and Weum S. 2012. Thermal Signals and Cutaneous Circulation in Physiological Research and Reconstructive Surgery. In: Diakides M, Bronzino JD, and Peterson DR, eds. Medical Infrared Imaging: Principles and Practices: CRC Press, 1-26. 
478 Pedersen BK, and Febbraio MA. 2008. Muscle as an endocrine organ: focus on muscle-derived

479

480

481

482

483

484

485

486

487

488

489

490

491

492

493

494

495 interleukin-6. Physiol Rev 88:1379-1406. 10.1152/physrev.90100.2007

Rifkind JM, Nagababu E, Dobrosielski DA, Salgado MT, Lima M, Ouyang P, and Silber HA. 2014. The effect of intermittent pneumatic compression of legs on the levels of nitric oxide related species in blood and on arterial function in the arm. Nitric Oxide 40:117122. 10.1016/j.niox.2014.06.007

Tripathi A, and Nadel ER. 1986. Forearm skin and muscle vasoconstriction during lower body negative pressure. Journal of applied physiology 60:1535-1541.

Vincent-Horta L, Sanders D, Martin J, and Beck D. 2015. Acute Effects of External Pneumatic Compression on Peripheral and Central Hemodynamics. The FASEB Journal 29:LB677.

Wallin BG, Sundlöf G, and Delius W. 1975. The effect of carotid sinus nerve stimulation on muscle and skin nerve sympathetic activity in man. Pflügers Archiv 358:101-110.

Williamson JW, Mitchell JH, Olesen HL, Raven PB, and Secher NH. 1994. Reflex increase in blood pressure induced by leg compression in man. J Physiol 475:351-357.

Wilson TE, Cui J, and Crandall CG. 2001. Absence of arterial baroreflex modulation of skin sympathetic activity and sweat rate during whole-body heating in humans. The Journal of physiology 536:615-623. 


\section{Table 1 (on next page)}

Participant characteristics for AIM1 and AIM2. 
Participant characteristics for AIM1 and AIM2.

\begin{tabular}{|c|c|c|c|c|c|c|}
\hline & \multicolumn{3}{|c|}{ AIM1 } & \multicolumn{3}{|c|}{ AIM2 } \\
\hline & Overall $(\mathrm{N}=18)$ & Males $(n=9)$ & Females $(n=9)$ & Overall $(\mathrm{N}=14)$ & $\operatorname{EPC}(n=7)$ & Sham $(n=7)$ \\
\hline Age, yrs & $25.5 \pm 4.7$ & $26.9 \pm 4.8$ & $24.1 \pm 4.3$ & $25.9 \pm 4.5$ & $25.6 \pm 4.0$ & $26.2 \pm 5.3$ \\
\hline height, $\mathrm{m}$ & $1.70 \pm 0.09$ & $1.76 \pm 0.8$ & $1.64 \pm 0.05 * *$ & $1.74 \pm 0.09$ & $1.75 \pm 0.07$ & $1.73 \pm 0.10$ \\
\hline body mass, $\mathrm{kg}$ & $75.1 \pm 16.0$ & $84.4 \pm 16.1$ & $65.7 \pm 9.6^{* *}$ & $82.9 \pm 14.9$ & $85.8 \pm 12.7$ & $79.9 \pm 17.3$ \\
\hline $\mathrm{BMI}, \mathrm{kg} / \mathrm{m}^{2}$ & $25.6 \pm 3.5$ & $26.9 \pm 3.5$ & $24.3 \pm 3.2$ & $27.2 \pm 2.7$ & $28.0 \pm 2.4$ & $26.4 \pm 2.9$ \\
\hline LA SBP, mmHg & $112 \pm 10$ & $117 \pm 9$ & $108 \pm 9 *$ & $117 \pm 10$ & $117 \pm 10$ & $117 \pm 12$ \\
\hline LA DBP, $\mathrm{mmHg}$ & $71 \pm 6$ & $76 \pm 4$ & $66 \pm 3 * *$ & $74 \pm 6$ & $77 \pm 3$ & $72 \pm 8$ \\
\hline $\mathrm{RA} \mathrm{SBP}, \mathrm{mmHg}$ & $111 \pm 11$ & $116 \pm 10$ & $105 \pm 10^{*}$ & $115 \pm 13$ & $117 \pm 11$ & $114 \pm 15$ \\
\hline $\mathrm{RA} \mathrm{DBP}, \mathrm{mmHg}$ & $71 \pm 8$ & $75 \pm 7$ & $66 \pm 8^{*}$ & $74 \pm 8$ & $77 \pm 5$ & $71 \pm 10$ \\
\hline
\end{tabular}

Data are mean \pm SD. BMI, body mass index; LA, left arm; SBP, systolic blood pressure; DBP, diastolic blood pressure; RA, right arm. ${ }^{* *},{ }^{*} p<0.05$ and $p<0.01$, respectively, for between sex comparisons (AIM1) 


\section{Table 2 (on next page)}

Popliteal artery flow-mediated dilation characteristics at baseline and after EPC in the treated and non-treated leg. 
Popliteal artery flow-mediated dilation characteristics at baseline and after EPC in the treated and non-treated leg

\begin{tabular}{lcc}
\hline & & EPC $(\mathrm{n}=18)$ \\
\cline { 2 - 3 } & PRE & POST \\
\hline Treated Popliteal Artery & $5.41 \pm 0.96$ & $5.49 \pm 0.94$ \\
Resting diameter, mm & $7.7 \pm 1.9$ & $7.4 \pm 1.9$ \\
Resting MBV, cm/s & $58.2 \pm 14.0$ & $55.3 \pm 15.2$ \\
Resting mean shear rate, $\mathrm{s}^{-1}$ & $300 \pm 119$ & $243 \pm 121^{*}$ \\
Reactive hyperemia MBV AUC, cm/s & $2245 \pm 897$ & $1878 \pm 1204^{*}$ \\
Reactive hyperemia shear rate AUC, A.U. & $0.11 \pm 0.04$ & $0.13 \pm 0.05^{*}$ \\
Absolute FMD, mm & & \\
Untreated Popliteal Artery & & $5.16 \pm 0.86^{\dagger}$ \\
Resting diameter, mm & $5.12 \pm 0.92 \dagger$ & $7.1 \pm 1.6^{*}$ \\
Resting MBV, cm/s & $8.0 \pm 1.2$ & $56.2 \pm 13.8^{*}$ \\
Resting mean shear rate, $\mathrm{s}^{-1}$ & $64.6 \pm 14.9$ & $266 \pm 121^{*}$ \\
Reactive hyperemia MBV AUC, cm/s & $331 \pm 129$ & $2121 \pm 1026^{*}$ \\
Reactive hyperemia shear rate AUC, A.U. & $2688 \pm 1147$ & $0.12 \pm 0.04^{*}$ \\
Absolute FMD, mm & $0.09 \pm 0.03$ & \\
\hline
\end{tabular}

Data are expressed as mean \pm SD. Mean blood velocity (MBV) and shear rate were measured continuously and resting measures are representative of the average of 2 minutes of baseline measurements, whereas area under the curve (AUC) measures are representative of values following cuff release until maximal dilation was observed ; FMD is flow mediated dilation expressed as absolute $(\mathrm{mm})$, relative $(\%)$, and normalized to the reactive hyperemic shear rate AUC. * and ${ }^{*}$, significantly difference between time points (pre vs. post; $(P<0.05$ and $P<0.01$, respectively); $\uparrow$, significantly different between legs at the same time point (e.g. post in compressed artery vs. post in non-compressed artery; $P<0.05$ ) 


\section{Table 3 (on next page)}

Average and regional mean skin temperature (MST) at baseline in each leg. 
Average and regional mean skin temperature (MST) at baseline in each leg

\begin{tabular}{cccccccc}
\hline & \multicolumn{3}{c}{ Right leg } & & \multicolumn{3}{c}{ Left Leg } \\
\cline { 2 - 4 } \cline { 6 - 7 } & Thigh MST & Lower-leg MST & Average MST & & Thigh MST & Lower-leg MST & Average MST \\
\hline EPC (n=7) & $30.6 \pm 1.1$ & $31.1 \pm 0.8$ & $30.9 \pm 1.0$ & & $31.0 \pm 0.9$ & $31.0 \pm 1.1$ & $31.0 \pm 1.0$ \\
Sham (n=7) & $30.6 \pm 0.5$ & $31.2 \pm 0.7$ & $30.9 \pm 0.6$ & & $30.5 \pm 0.5$ & $31.2 \pm 0.7$ & $30.9 \pm 0.6$ \\
\hline
\end{tabular}

$\begin{array}{llll}\text { Sham }(\mathbf{n}=7) & 30.6 \pm 0.5 & 31.2 \pm 0.7 & 30.9 \pm 0.6\end{array}$ 


\section{Figure 1}

Representative thermograms.

Pictured are thermograms of a participant's lower limbs (A) after equilibration to environmental conditions (i.e. baseline [PRE]), (B) during external pneumatic compression (EPC; T15), and (C) immediately post-EPC (R0).

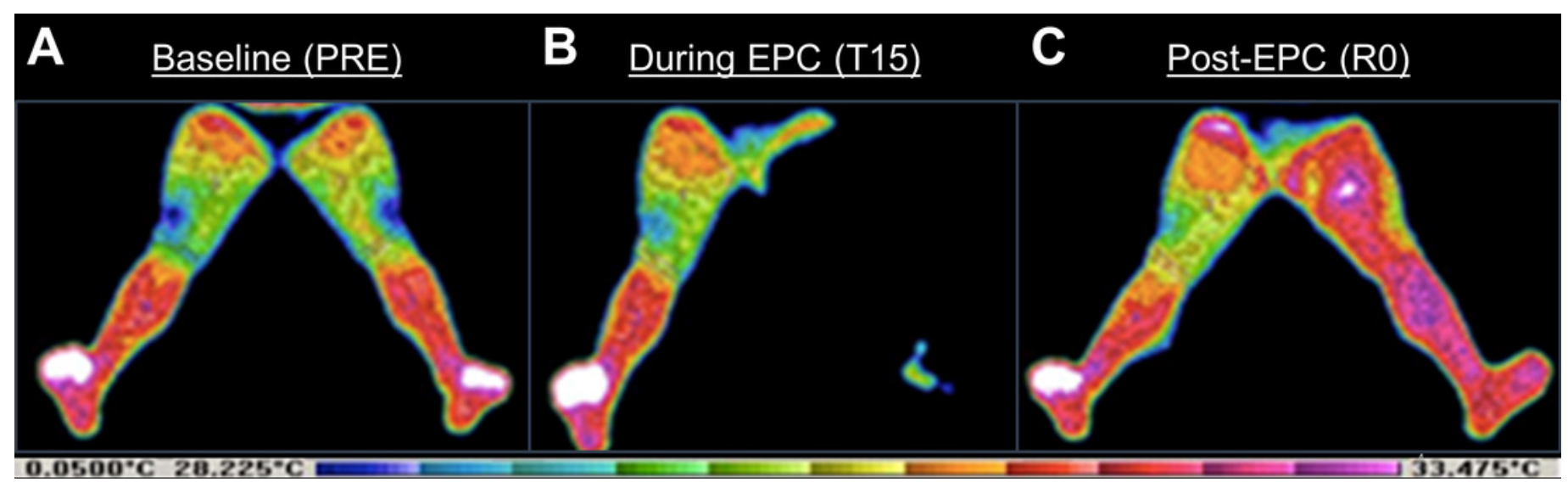


Figure 2

Popliteal artery blood flow and flow mediated dilation (FMD) characteristics in both legs before (PRE) and 30-min after (POST) a bout of unilateral external pneumatic compression (EPC).

A) maximal ( $\max )$ popliteal artery blood flow during reactive hyperemia, B) total popliteal artery blood flow during reactive hyperemia, C) relative FMD (\%), and D) FMD normalized to shear rate area under the curve to peak dilation ( $\mathrm{nFMD}$ ). Data are presented as mean $\pm \mathrm{SD}$ for each leg (treated vs. untreated) at each time point (PRE vs. POST). ANOVA results are presented within each panel with significant main effects emphasized in bold.

A

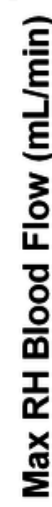

C

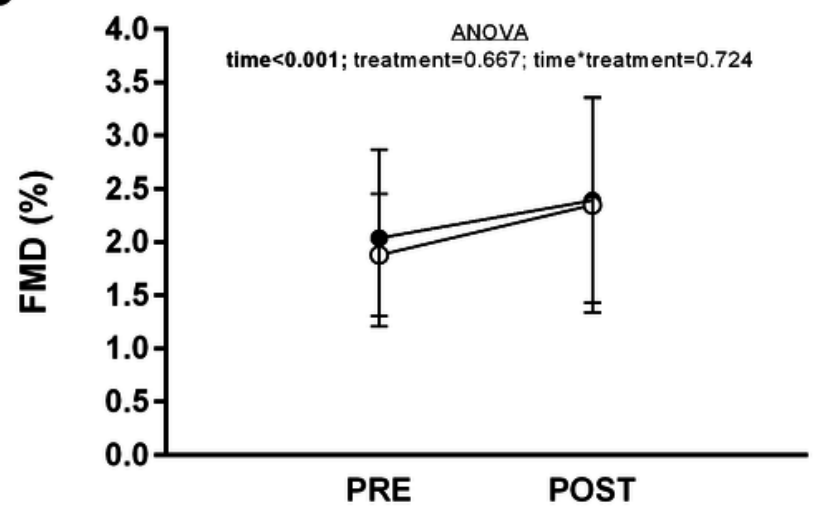

B

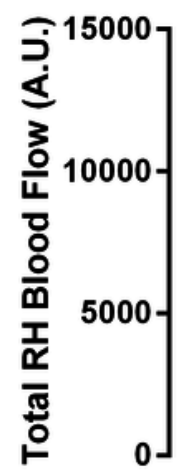

time $=0.151 ;$ treatment $=0.999 ;$ time ${ }^{*}$ treatment $=0.431$
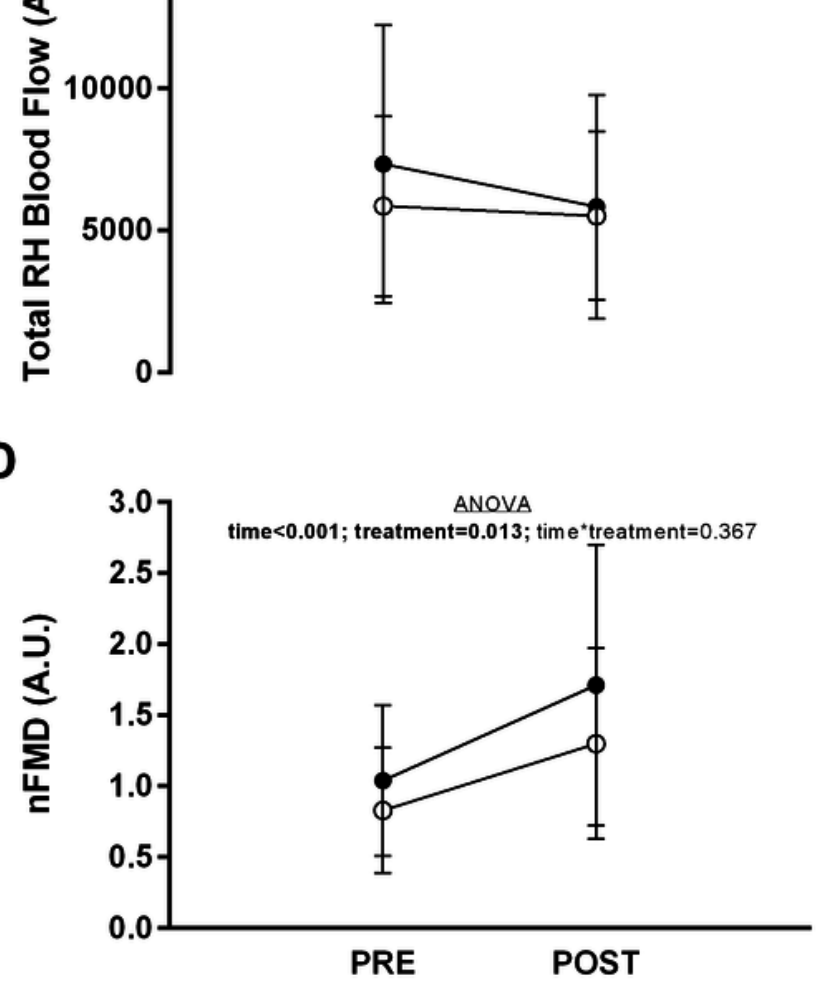


\section{Figure 3}

Change $(\Delta)$ from baseline in mean skin temperature (MST) at the mid-point (T15) and immediately following (R0) external pneumatic compression (EPC)/sham treatment.

Data are presented as individual values and mean $\pm 95 \%$ confidence interval. Panels illustrate the observed A) time*treatment (EPC vs. Sham; data collapsed across regions) and B) time*region (thigh vs. lower-leg; data collapsed across treatments) interactions for MST at T15, and C) time*treatment*leg (treated vs. untreated; data collapsed across regions) and D) time*region (data collapsed across legs and treatments) interactions for MST at R0. *, significant change from baseline $(p<0.0167)$ and $\phi$, significantly different between factors $(p<0.0167)$. For panel $c: t$, significant change from baseline $(p<0.0125)$ and different superscript letters between groups indicate significant differences $(p<0.008)$. 

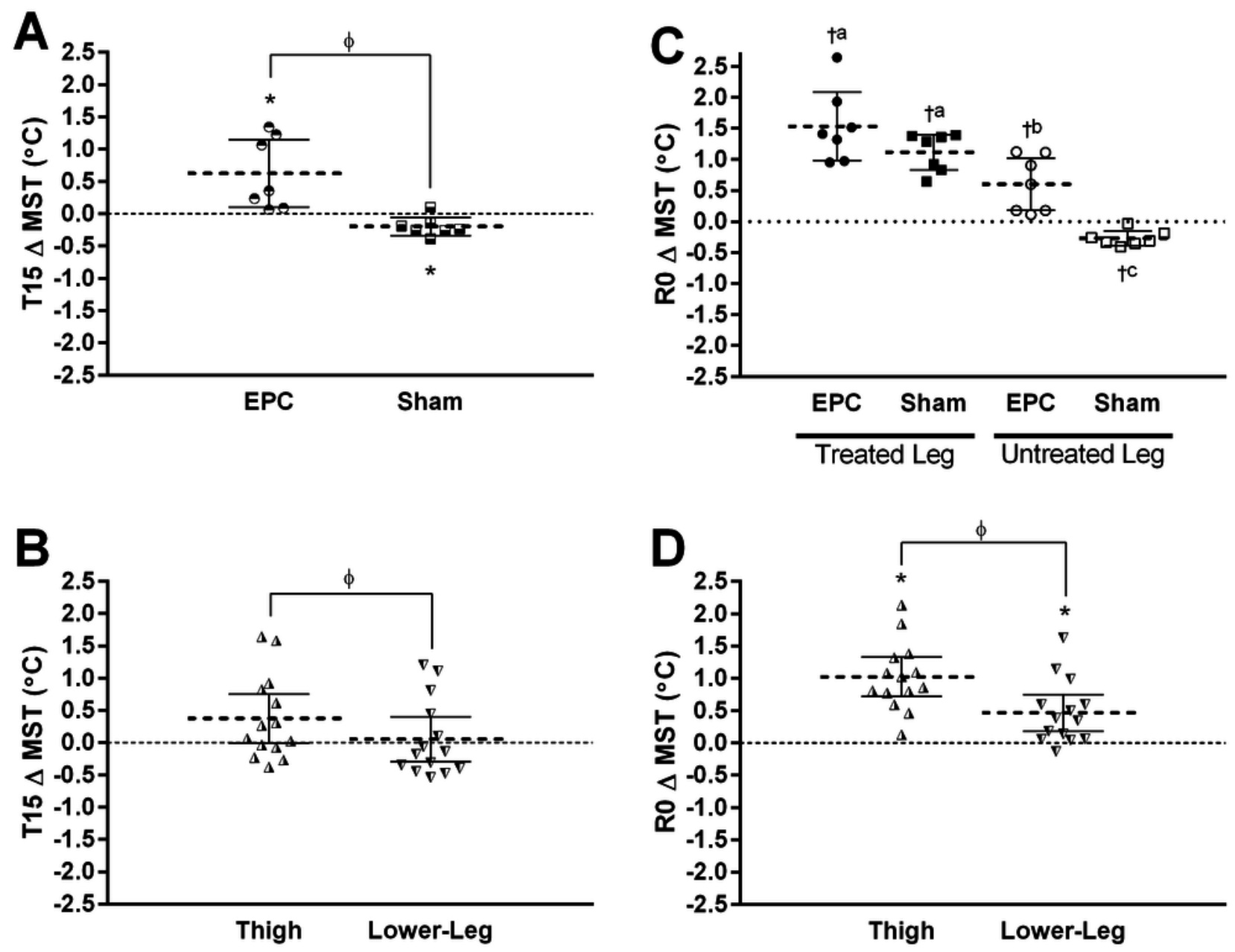


\section{Figure 4}

Change from baseline in mean skin temperature (MST) at 30- (R30) and 60-min (R60) following external pneumatic compression (EPC)/sham treatment.

Data are presented as individual values and mean $\pm 95 \%$ confidence interval. Panels illustrate the observed A) time*treatment (EPC vs. Sham; data collapsed across regions and legs), B) time*region (thigh vs. lower-leg; data collapsed across treatments and legs), and C) time*leg (treated vs. untreated; data collapsed across treatments and regions) interactions for MST at R30 and D) time*treatment, E) time*region, and F) time*leg interactions for MST at R30. *, significant change from baseline $(p<0.0167)$ and $\phi$, significantly different between factors $(p<0.0167)$. 

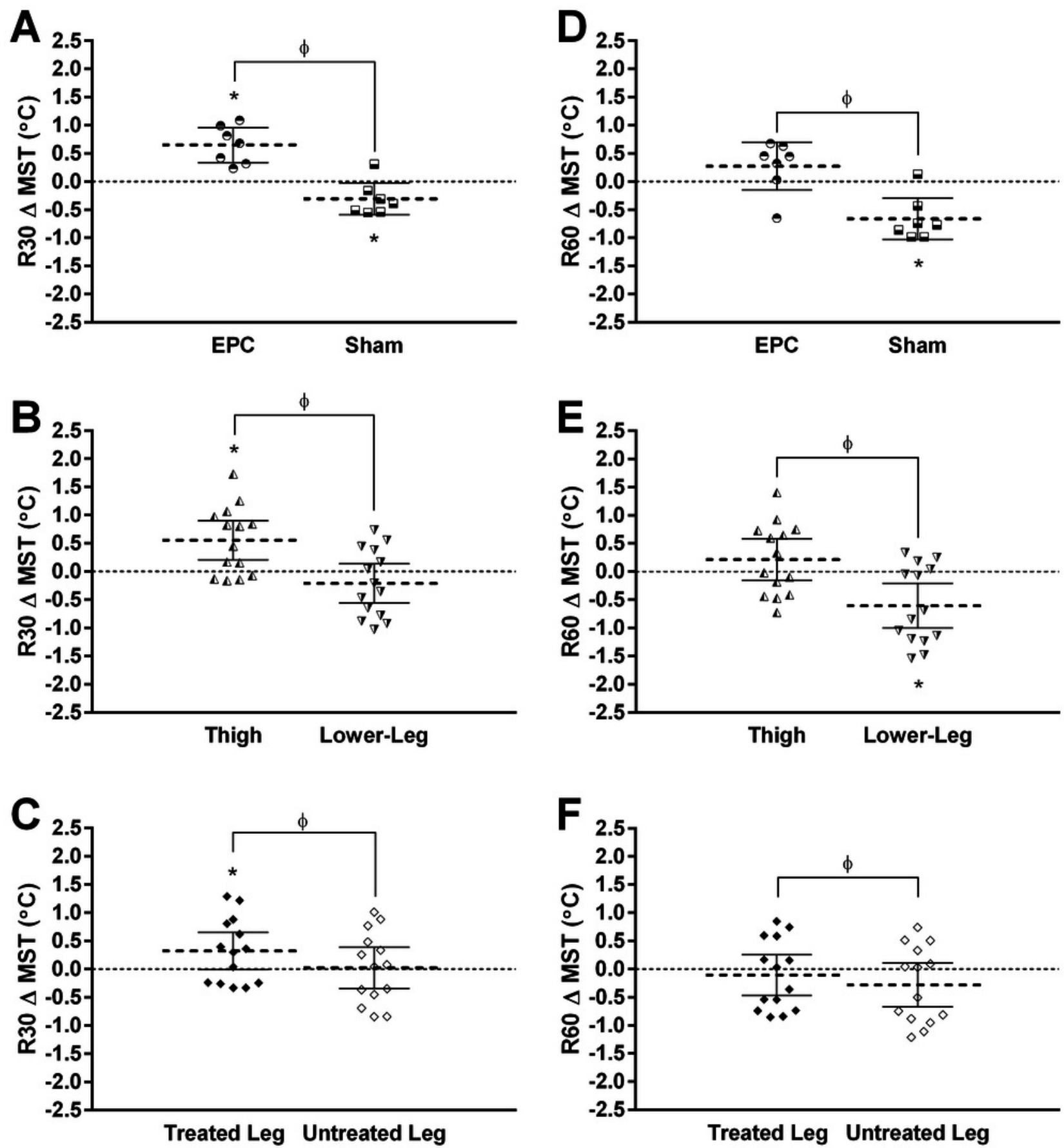